\title{
DAS ÄGYPTISCHE PSEUDOPARTIZIP ${ }^{1}$
}

1. 1889 hat A. Erman unter dem Titel »Eine neue Art der ägyptischen Conjugation « einen epochalen Aufsatz veröffentlicht, ${ }^{2}$ in dem er zum ersten Mal eine Reihe von bis dahin nicht als zusammengehörig erkannten Formen zu einem einzigen Paradigma zusammengestellt hat. Wie schon der Titel sagt, hat Erman diese Form als eine besondere Art des finiten Verbs bestimmt und mit dem (west)semitischen Perfekt verglichen, womit in der Tat eine auffallende Ähnlichkeit besteht. Es ist für die Behandlung dieser Form in der Ägyptologie von nicht zu unterschätzender Bedeutung gewesen, daß Erman damals das westsemitische Perfekt heranzog, eine Form, die unbestritten ein finites Verb ist, aber nicht den viel näher und deutlicher verwandten akkadischen Stativ, an dessen verbaler Natur schon eher Zweifel möglich sind. ${ }^{3} \mathrm{Zu}$ dieser Zeit stand eben die Erforschung des »Assyrischen « noch in den Anfängen. ${ }^{4}$ Der Vergleich mit dem Perfekt hat sich sonderbarerweise auch noch viel später gehalten: So verweist Gardiner ${ }^{5}$ zwar auf den akkadischen "permansive«, gibt der ägyptischen Parallelform aber dennoch den völlig irreführenden Namen »old perfective «. ${ }^{6}$

' Abkürzungen nach Lexikon der Ägyptologie, Bd.VII, p. XIVff. Zusätzlich EAG = Edel, Altägyptische Grammatik, AnOr 34/39, 1955/64; GEG = Gardiner, Egyptian Grammar, Oxford 1957; LGE = Lefebvre, Grammaire de l'égyptien classique, BdE 12, 1955.

2 ZÄS 27, 65-84.

${ }^{3}$ Von Soden, Grundriß der akkadischen Grammatik, AnOr 33/47, 1969, § 77a: »Der Stativ nimmt unter den »Tempora « ... eine Sonderstellung ein, ... da er eigentlich ein konjugiertes Nomen ist « [Hervorhebung im Original]. Genaueres s.u., §9.

${ }_{4}^{4}$ Aber auch in der 4. Auflage seiner Ägyptischen Grammatik von 1928 (§ 325) zieht er zum Vergleich nur das arabische Perfekt heran!

5 GEG $\& 309$, Obs. 1 .

${ }^{6}$ Es ist auch sonst sehr auffällig, daß die enge Verwandtschaft des Pseudopartizips mit dem akkadischen Stativ überall pflichtschuldigst registriert worden ist, dennoch die Vorstellung vom »Perfekt « zu dominieren scheint, obwohl sich ja ein "Perfekt « erst ganz sekundär in einer innersemitischen Entwicklung herausgebildet hat. Man vergleiche aus neuester Zeit z.B. Kammerzell, GM 117/118, 1990, p. 181ff, der den adverbiellen Gebrauch des Pseudopartizips/Stativs »Pseudopartizip« nennt, den prädikativen der 1. Person aber $\gg$ Perfekt «.

Rätselhaft ist mir Schenkels Haltung: Er zitiert zwar zustimmend Buccellatis Feststellung, daß der akkadische »Stativ« nichts weiter als ein Nominalsatz sei (Die altägyptische Suffixkonjugation, ÄA 32, 1975, p. 71-2; zum »Stativ« s. unten, § 9), hält aber trotzdem an seiner alten These vom Medium/Perfekt fest (OrNS 40, 1971, 301-16; s. zuletzt AfO 35, 1988, 238), die doch gerade durch Buccellatis Schlußfolgerungen ins Herz getroffen wird. 
Man kann vermuten, daß Ermans Überzeugung, die ägyptische Form sei (ursprünglich) ein finites Verb gewesen, ganz wesentlich durch den Vergleich mit dem westsemitischen Perfekt, der »Suffixkonjugation «, zustandegekommen ist. Hätte er schon den akkadischen Stativ gekannt, wäre er wohl keineswegs so sicher gewesen.

In der Zeit vor Erman hatte man die Formen des Pseudopartizips noch auf verschiedene Paradigmen verteilt: Ein Teil wurde als finite Verben erklärt, die Pseudopartizipien in der 3. Person galten dagegen als Partizipien. ${ }^{7}$

Erman hält nun das Pseudopartizip für eine »besondere alte Conjugation des Verbum finitum « (p. 77), die »ursprünglich nicht (die) participiale Bedeutung hat « (p. 76). Er gibt aber zu, daß schon im Mittleren Reich »die Ägypter sie [diese Konjugation] wirklich als eine besondere Art Participien empfunden haben « (p. 77-8), weshalb er dann auch den Namen »Pseudopartizip « dafür vorgeschlagen hat.

Diese letztere Einschränkung, daß das Pseudopartizip im Mittelägyptischen tatsächlich eine Art Partizip ist, sich also aus einem finiten Verb dazu entwickelt habe, ist dann von Ermans Nachfolgern nicht mehr beachtet worden. In den neueren Grammatiken (und sonstigen Arbeiten) wird das Pseudopartizip ${ }^{8}$ überall als reine Verbalform eingeführt und (Pseudo)Partizip als mehr oder weniger sinnentleerter Name behandelt. Bezeichnend ist Sottas' ironische Bemerkung: ${ }^{9} \gg$ Le vocable a un mérite appréciable. S'il est plein de son, il est à peu près vide de sens et, à tout prendre, un non-sens prête moins aux confusions qu'un contresens. «

2. Zu Bildung, Funktion und Bedeutung des Pseudopartizips im Altund Mittelägyptischen läßt sich kurz zusammengefaßt folgendes sagen:

- Es kann von (fast) allen Verbalstämmen gebildet werden (aber nicht von [grundsätzlich] allen Nomina wie der Stativ im Akkadischen).

${ }^{7}$ Für die alte Auffassung vgl. man Piehls polemischen Artikel »A propos du terme 'Pseudoparticipe' qu'à tort on a voulu introduire en égyptien «, Sphinx V, 1902, 27-31, worin Ermans Aufsatz als »tout-à-fait dépourvu(e) de sens commun et d'intérêt scientifique « (p. 28) dargestellt wird.

${ }^{8}$ Oder der »Stativ«: Merkwürdigerweise hat man vom Akkadischen ausgerechnet den Namen übernommen, obgleich er dort so unpassend wie für das Ägyptische ist, s. Huehnergard, JNES 46, 1987, 232, n. 64.

${ }^{9}$ RecTrav 40, 1923, 77-8; zitiert von Polotsky, Collected Papers, Jerusalem 1971, p. 337 , n. 32 . 
- Seine hauptsächlichen Funktionen sind:

a) Als eine Art »Umstandssatzerweiterung « (wie es zur Zeit verstanden wird), jeweils auf einen Aktant im Satz bezogen (Subjekt, Objekt, Präpositionalphrase), z.B. n sdr z ḥqrw r dmj.j »kein Mann verbrachte die Nacht in meiner Stadt, indem er hungrig war ${ }^{10}$.

aa) Als Sonderfall davon kann das Pseudopartizip im »virtual relative clause $\ll$ nach indeterminiertem Bezugswort gelten (GEG § 317).

b) Als »Umstandssatzerweiterung « mit vorausgehendem Substantiv (»eigenem Subjekt«), z.B. n't $\mathrm{m}$ hd jn hm.f jb.f $3 w(w) » s e i n e$ Majestät zog nach Norden, indem sein Herz froh war «" ${ }^{11}$.

bb) Als Sonderfall die (seltenen) Belege, wo Substantiv + Pseudopartizip von einer Präposition abhängig sind: »Ich gab Korn an Jwnj und Hf3t m-ht Jw-m-jtrw s inht(j) nachdem J. versorgt war ${ }^{12}$, s. GEG $§ 327$ sowie Satzinger, MDAIK 22, 1967, 102.

c) Als Prädikat eines (Haupt)Satzes, auf das Subjekt folgend (oft nach bestimmten Partikeln).

d) Als selbständiges Prädikat ohne vorhergehendes Subjekt, auf die 1. Person Sg. beschränkt. ${ }^{13}$

Der angebliche selbständige Gebrauch des Pseudopartizips in der 2. und 3. Person als »Optativ« bzw. in »exclamatory use « wird hier nicht weiter betrachtet, da diese Fälle wohl ausnahmslos redensartliche Verkürzungen (u.ä.) sind (vgl. GEG § 313, Obs.; Westendorf, Grammatik der medizinischen Texte, § 190).

— Die Bedeutung eines Verbalstammes im Pseudopartizip ist passiv bei transitiven Verben, resultativ bei intransitiven und »deskriptiv « bei Zustands/Eigenschaftsverben. In der Funktion d) haben in alten Texten transitive Verbstämme aktive Bedeutung (s.u., § 10).

\section{Zunächst soll Fall a) näher betrachtet werden:}

Hier gibt das Pseudopartizip eine nähere Qualifikation eines der Aktanten des Verbs (Subjekt/Agens, Objekt, Präpositionalergänzung),

10 SETHE, Lesestücke, p. 79, Z. 15-6.

11 Urk IV, 5, 12-3.

12 Kairo CG 20001, Z. 6.

13 Die Buchstaben a) - d) werden im folgenden laufend verwendet, um auf die Funktionen des Pseudopartizips zu verweisen. 
aber es ist deshalb kein Attribut dieses Aktanten, sondern bezieht sich zugleich auf das Verb, indem es dieses in gewisser Weise ebenfalls qualifiziert bzw. einschränkt: $n$ sdr $\mathrm{z} \operatorname{hqr}(w) r$ dmj.j »kein Mann verbrachte die Nacht hungrig in meiner Stadt «. In diesem Beispiel wird das Subjekt des Verbs (z) näher beschrieben und zugleich der Verbalvorgang: Es handelt sich nicht einfach um ein »(nicht) die-Nachtverbringen «, sondern um ein $»\left(\right.$ nicht) hungrig-die-Nacht-verbringen $« .{ }^{14}$

Die Behauptung, das Pseudopartizip bezöge sich grundsätzlich zugleich auf ein Verb und einen Verbaktanten, ist etwas einzuschränken: In ganz seltenen Fällen kann es sich auch auf ein nichtverbales Prädikat (sowie eine Konstituente des Nichtverbalsatzes) beziehen. Aus den Pyramidentexten vergleiche man:

§ 195c: nfr w(j) hr.t ḥtp.t(j) m3(wj).tj rnpw.tj »wie schön ist dein Gesicht, wenn du zufrieden, erneuert und verjüngt bist «;

$\S 1621 \mathrm{a} / \mathrm{b}: \underline{t w t} \mathrm{nb}$ t3 $\mathrm{r}$ dr.f shm.tj m psdt ntr nb jst »du bist der Herr des ganzen Landes, indem du Macht hast über die Neunheit und jeden Gott $\ll$.

Das sind aber (zumindest aus den PT) schon die einzigen einigermaßen eindeutigen Belege (und auch hier ist optativische Bedeutung nicht restlos auszuschließen). In anderen möglichen Fällen:

§ 685a ḥr ḥr.k [als Befehl] p3hd.tj;

$\S 685 b$ hr hr.k " $\quad$ st $3 . t j$;

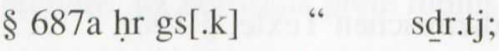

$\S * 1831 \mathrm{~d} / \mathrm{e}=\mathrm{Nt} 368:$ ḥ3 NN twt ntr '3 wr.tj dbn.tj m dbn ḥ3 nbwt;

$\S 1729 \mathrm{a} / \mathrm{b}:$ j3jw jr.k <j>3 wr jr.k j3j.tj šrt.k ḥr sțj jht-wtt

wäre optativische Bedeutung ebenso passend oder sogar besser.

Von diesen und ganz seltenen ähnlichen Beispielen abgesehen aber werden Pseudopartizipien dieser Art in nahezu allen Fällen auf ein Verb und einen Aktanten bezogen sind: Das Verb kann dabei auch nominalisiert sein, z.B. Urk IV, 1229, 14: njswt pw 'h3 w'w »er ist ein König, der allein kämpft « bzw. seinerseits im (prädikativen) Pseudopartizip stehen, z.B. Schiffbr. 7: jzwt.n jj.t(j) ¿d.t(j) »unsere Mannschaft ist

${ }^{14}$ Das Pseudopartizip dieses Beispiels war auf das Subjekt des Verbs bezogen. Beispiele, wo es auf das Objekt bezogen ist, z.B. in Westc., 7,14-5: gmj.n.f sw sdr(w), auf eine präpositionale Ergänzung z.B. in Sinuhe B 199-200: spr.n wd pn r.j 'ḥ'.kwj m hrr-jb wḥt.j. 
unversehrt zurückgekommen«. Gardiners Beispiel Sinuhe B 193-4 (GEG § 314, Nr.8) ist unvollständig zitiert; er hat das Verb, worauf sich das Pseudopartizip bezieht, weggelassen:

jrj.tw n.k šms-wḋ hrww zm3-t3

- wj m nbw

- tp m hssbd

- pt hr.k

- dj.t(j) m mstpt

- jḥw hr jtḥ.k

- šm w hr-ḩ̧t.k.

Das dj.t(j) ist hier wieder zugleich auf jrj und auf .k bezogen!

Eine solche Konstruktion, »attributiv « auf einen Verbaktanten und zugleich »adverbial « auf das Verb bezogen, wird in der Grammatik der klassischen Sprachen (zumindest im deutschsprachigen Bereich) gewöhnlich »Prädikativum « genannt. Es handelt sich — sachlich und terminologisch - um eines der heikelsten Gebiete der Grammatik, und man hat eine ganze Reihe von Bezeichnungen für die Erscheinung, ohne daß es irgendeinen Konsens gäbe: »complément prédicatif « ${ }^{15}$, »secondary predicate $\ll^{16}$, »prädikatives Attribut $\aleph^{17}$ u.a.m. »Prädikativum « ist hier nur mit großem Vorbehalt gewählt, da es sehr mißverständlich ist: In den meisten Grammatiken des Deutschen z.B. ist damit auch das »Prädikatsnomen « (z.B. »er ist gro $\beta$ «) gemeint. ${ }^{18}$

Lefebvre ${ }^{19}$ hat auf einige Fälle verwiesen, wo es schwierig ist, zwischen Pseudopartizip und passivem Partizip zu unterscheiden. Das Problem, in vielen Fällen schwer vom Attribut zu unterscheiden zu sein, ist typisch für Prädikativa, vgl. den systematischen Überblick bei M. Kienpointner, Kontrastive Darstellung lateinischer und deutscher Prädikativa im Rahmen der Dependenzgrammatik, Innsbruck 1985, p. 17-20.

5 Vergote, Fs Grapow, p. 339.

${ }^{6}$ Polotsky, Grundlagen des koptischen Satzbaus, Atlanta 1990, p. 226.

17 H. Bubmann, Lexikon der Sprachwissenschaft, Stuttgart 1983, p. 126.

18 Diese Zusammenfassung von »Prädikatsnomen « und »Prädikativum « ergibt sich zwangsläufig, wenn man das Hilfsverb »sein « als »Vollverb « behandelt.

Ich unterscheide im weiteren konsequent zwischen "prädikativ« (=als/wie ein Prädikat) und »prädikativisch « (als/wie ein Prädikativum).

19 LGE \& 355. 
Diese Funktion des Pseudopartizips ist 1955 von J. Vergote in einem fundamentalen Aufsatz dargestellt worden. ${ }^{20} \mathrm{Er}$ gibt dort zunächst einen Überblick über die verschiedenen Möglichkeiten prädikativischer Ergänzungen im allgemeinen (p. 341-44), und bespricht dann auf p. 345-6 (I, II) und p. 349 (IV) die hier kurz beschriebene Funktion a) des Pseudopartizips. ${ }^{21}$ Ich kann hier auf weitere Beispiele verzichten, die man bei Vergote ja reichlich findet.

Als Vergote seinen Aufsatz schrieb, galt in der Ägyptologie Polotskys Theorie von den substantivierten Verbalformen, die häufig als Subjektssätze auftreten, noch als ziemlich exotisch. Das hat sich inzwischen geändert: Heute würden die meisten ein jrj.n.j hrw 3 w j.kwj (Vergote, Bsp. 1) als »daß ich 3 Tage verbrachte, war (indem ich) allein (war)« analysieren und ein prr.j h.zj.kwj m "h (Vergote, Bsp. 5) als »daß ich aus dem Palast komme, ist belohnt «. Das ist aber kein Einwand gegen Vergotes überzeugende Interpretation: Auch im Deutschen etwa kann man ein Prädikativum, z.B. »ich traf ihn schlafend an « [auf das Objekt bezogen] zum Prädikatsnomen machen und das Hauptverb in einem Subjektssatz einführen: »Daß ich ihn antraf, war schlafend «.22 Obwohl jetzt »war schlafend « die Prädikatsgruppe ist, ändert sich nichts daran, daß »schlafend « sich auf das Objekt des Verbs bezieht sowie adverbial auf das Verb selbst!

Die Annahme einer prädikativischen Funktion des Pseudopartizips steht also dem Glauben an die »emphatischen « Formen und Funktionen Polotskys nicht im Weg. Sie würde allerdings sehr wohl eine Stütze für die These sein, daß es sich bei diesen Konstruktionen um sekundäre Entwicklungen handelt und das Verb das ursprüngliche Prädikat des Satzes ist. ${ }^{23}$

4. Auch den Fall b), »Umstandssatzerweiterung « mit vorausgehendem Substantiv, hat Vergote völlig überzeugend den prädikativischen

20 »La fonction du pseudoparticipe «, Fs Grapow, Berlin 1955, p. 338-61. Er hat allerdings für die prädikativische Funktion unglücklicherweise den Terminus »nexus « gewählt, der sonst anders gebraucht wird.

${ }^{21}$ Es ist mir aber nicht klar, warum er seine Gruppe IV (p. 349) zu den Fällen von »casus absolutus « stellen möchte und nicht einfach zu den anderen Verbaktanten Subjekt und Objekt: Auf jeden Fall halte ich diese Eingruppierung nicht für korrekt. Zu weiteren Änderungsvorschlägen zu Vergotes Systematik s.u.

${ }^{22}$ Das ist sicher kein sonderlich elegantes, aber doch grammatisches Deutsch.

$23 \mathrm{Vgl}$. in diesem Sinne auch ALLEN in: Essays on Egyptian Grammar, New Haven 1986, p. 16-7. 
Konstruktionen zugewiesen (p. 347-9: III). ${ }^{24}$ Das gilt zumindest für einen Teil der Belege: Fälle wie das oben angeführte ( $n^{\complement} t \mathrm{~m}$ hd jn ḥm.f) $j b . f 3 w(w)$ sind exakte Parallelen zu den in den klassischen Sprachen so häufigen absoluten Partizipialkonstruktionen wie dem »ablativus absolutus « im Lateinischen oder dem GcP im Griechischen. ${ }^{25}$ Ebenso gehören die (selteneren) von Präpositionen abhängigen Belege für Substantiv + Pseudopartizip (GEG § 327) hierher; sie verhalten sich genau entsprechend einem »ab urbe condita « oder »ante Christum natum «, wie Vergote ausführt. Das ist um so einleuchtender, als es die Alternative, einen von einer Präposition abhängenden Adverbialsatz im Mittelägyptischen ja gar nicht gibt (vgl. GEG § 327)! ${ }^{26}$

Obwohl Vergotes Ausführungen im Grundsätzlichen sicher richtig sind, möchte ich doch seine Klassifizierung der Belege etwas modifizieren: ${ }^{27}$

- Seine Gruppe IV (p. 349) gehört vor III (Prädikativum auf Subjekt, Objekt oder präpositionale Ergänzung bezogen).

- Die Subjektsgruppe (I) ist zu teilen, je nachdem, ob das Prädikativum aus einem Wort besteht $(\mathrm{A}+\mathrm{B})$ oder ob es seinerseits Ergänzungen zu sich nimmt (C). Im letzten Fall ist die Beziehung zum Verb offenbar loser.

- Gruppe III enthält nicht nur »absolute « Konstruktionen, d.h. Substantiv + Prädikativum (wie 27 und 30), sondern eine ganze Reihe von Fällen, die in II bzw. IV einzuordnen wären (z.B. 25, 26, 29, 3133; die Fälle 34-6 sind auch aus anderen Gründen problematisch ${ }^{28}$ ). Beleg 28 ist nicht adverbial auf ein Verb bezogen, gehört also nicht hierher: Das Pseudopartizip von 28 ist prädikativ, nicht prädikativisch, s.u. § 7. Das heißt, die Belege für Funktion b) sind in zwei Gruppen aufzuspalten: In einem Teil handelt es sich um Prädikativa

${ }^{24} \mathrm{Ob}$ seine Benennung »Nexus-subjunctum « sehr glücklich ist, darf man aber bezweifeln.

${ }^{25}$ Auch im Deutschen gibt es - in etwa - entsprechende Konstruktionen, die in den Grammatiken meist recht stiefmütterlich als »verkürzte Nebensätze «, »satzwertige Wortgruppen « u.ä. behandelt werden, z.B. »in der Stadt angekommen, suchte er sofort einen Arzt auf «. Die meisten Grammatiken sind sich einig, daß in diesen Fällen ein Partizip (»seiend/habend «) zu ergänzen ist.

${ }^{26} \mathrm{Im}$ Altägyptischen gibt es zwei dubiose Fälle in den Pyramidentexten, die auch anders verstanden werden können, s. EAG § 922, Anm.

${ }^{27}$ Die Zahlen beziehen sich auf die durchnumerierten Beispiele von Vergote.

${ }^{28}$ Vermutlich handelt es sich gar nicht um Pseudopartizipien. Zu Nr.35 s. WESTENDORF, Der Gebrauch des Passivs in der klassischen Literatur der Ägypter, Berlin 1953, p. 74-5. 
in »absoluten « Konstruktionen, in dem anderen um adverbiell gebrauchte Gliedsätze mit adverbialem Prädikat. ${ }^{29}$

- Bei den auf das Objekt oder eine andere Ergänzung bezogenen Prädikativa wäre zunächst zu unterscheiden, ob sie von der Valenz des Verbs gefordert $\operatorname{sind}^{30}$ wie z.B. in den Belegen 16, 18, 19, 22-24, und bei dem Rest (der den eigentlichen participia coniuncta entspricht) dann zu trennen zwischen aus einem Wort bestehenden Prädikativa (z.B. 17, 20, 21) und denen, die ihrerseits eine Ergänzung zu sich nehmen (z.B. 26, 29, 31-33: hier ist wieder die Beziehung zum Verb loser).

Vergotes Beschreibung der Funktion des Pseudopartizips als Prädikativum und sein Vergleich der Funktion b) mit den absoluten Partizipialkonstruktionen ist weitgehend unbeachtet geblieben. Möglicherweise hängt das damit zusammen, daß er, wie im folgenden gezeigt wird, mit seiner Analyse etwas zu weit gegangen ist und auch klare Fälle von prädikativem Gebrauch als prädikativisch eingestuft hat.

5. Der Fall aa) (Pseudopartizip im Relativsatz mit indeterminiertem Bezugswort) wird von Vergote ebenfalls zur Sprache gebracht (p. 35761), aber hier leuchtet mir seine Interpretation nicht ein. Er unterscheidet zunächst — sehr mit Recht — generell zwei unterschiedliche Gebrauchsweisen eines Attributs, nämlich als »épithète déterminative « und als »épithète descriptive «. ${ }^{31}$ Das Pseudopartizip stehe in dieser Konstruktion in komplementärer Distribution mit dem Partizip: Das Partizip sei determinativ (spezifizierend), das Pseudopartizip deskriptiv (charakterisierend). Das kann aber nicht richtig sein: Erstens gibt es

${ }^{29}$ Im Einzelfall kann eine Unterscheidung problematisch werden: Die Mittelstellung der Prädikativa zwischen Satzglied und Gliedsatz ist ja (für die klassischen Sprachen) oft betont worden.

${ }^{30}$ Was nicht unbedingt gleichbedeutend mit »nicht weglaßbar « ist! Kienpointner, op. cit., etwa teilt die Prädikativa in valenzgeforderte »Prädikativergänzungen « und freie »Prädikativangaben « ein (p. 4), unterscheidet bei den Prädikativergänzungen aber wiederum zwischen obligatorischen und fakultativen, also weglaßbaren (p. 11-2).

Zum Unterschied zwischen »freien « und valenzgeforderten Prädikativa s.a. POLOTSKY, Grundlagen des koptischen Satzbaus, II, 228, der Verben mit entsprechender Valenz als Verben »unvollständiger Prädikation « bezeichnet.

31 H. SeILER, Relativsatz, Attribut und Apposition, Wiesbaden 1960, p. 19ff. benennt denselben Unterschied »spezifizierend « vs. »charakterisierend «, ihm folgend ebenso SCHENKEL, MDAIK 22, 1967, 74f. und JUNGE, Syntax der mittelägyptischen Literatursprache, Mainz 1978, 68f. 
unzählige »charakterisierende « Partizipien: Man denke an die lobenden Beiworte in den biographischen Texte, wo sehr häufig Partizipien vorkommen; dennoch sind diese Beiworte in keiner Weise einschränkend »spezifizierend «! Zweitens gibt es ungekehrt ebensogut spezifizierende Pseudopartizipien, z.B. in Vergotes Beispiel 117 (p. 360) = Ebers 49,1-2: št jzt snwh.tj ḥr mrḥt, wo seine Erklärung eine reine Ausrede ist. Vergleichbare Fälle sind in den medizinischen Texte nicht selten und praktisch immer spezifizierend! Vergotes Lösung ist also in diesem Punkt nicht zutreffend. ${ }^{32}$

Es wäre hingegen sehr wohl möglich, daß es sich beim Pseudopartizip im Relativsatz wiederum um eine prädikativische Gebrauchsweise handelte. Da diese Verwendung des Pseudopartizips nur ein Sonderfall innerhalb der Relativsätze ist, empfiehlt es sich, die Relativsätze insgesamt zu betrachten.

Es ist in der ganzen ägyptischen Sprachgeschichte so, daß nach indeterminiertem Bezugswort Umstandssätze als Relativsätze dienen. Noch im Koptischen hat man пршмє eтnanoyq vs. oүpшme ENANOYq. Das hat sich auch, als das Arabische in Ägypten dominierte, nicht geändert: Arabisch (Hochsprache und Dialekt) verhält sich in dieser Hinsicht wie das Ägyptisch-Koptische. ${ }^{33}$ Für das Mittelägyptische vgl. man GEG § 196-8; 317. Man kann diese merkwürdige Erscheinung m.E. folgendermaßen erklären:

»Determiniertheit « bzw. »Indeterminiertheit « besagt als pragmatische Kategorie, ob ein Nomen (genauer gesagt dessen Referent) dem Sprecher/Hörer bekannt (im Sinne von bereits »präsentiert «) ist (determiniert) oder nicht (indeterminiert). ${ }^{34}$ Für Sprachen mit Artikel hat H. Weinrich diese Tatsache weiter verdeutlicht: ${ }^{35}$ Der bestimmte Artikel verweist zurück auf die Vorinformation des Textes (d.h. auf bereits

${ }^{32}$ Sein betreffender Abschnitt enthält auch sonst einige Schwachpunkte: So ist die Verbindung "la femme d'un prêtre " selbstverständlich indeterminiert (so wie im Deutschen »die Frau eines Priesters « = »eine Priesterfrau «). Bei Genetivverbindungen bestimmt das Nomen rectum den Determinationsgrad der Gesamtverbindung, vgl. WEINRICH, "Textsyntax des französischen Artikels « in: Sprache in Texten, Stuttgart 1976, p. 197-8. Eine Verbindung des Typs »une femme d'un prêtre « kommt nach Weinrich so gut wie nie vor.

${ }^{33} \mathrm{Zu}$ einer rezenten Bemühung um die Relativsätze im klassischen Arabisch s. Faleh Alajmi, Relativsatz im Klassischarabischen, Diss. Gießen 1988.

${ }_{34}$ Bußmann, Lexikon der Sprachwissenschaft, p. 81.

35 Op. cit., p. 186-98. 
Präsentiertes) bzw. auf allgemein Bekanntes (das keiner besonderen Einführung bedarf), der unbestimmte Artikel verweist voraus auf nachfolgende Information (noch nicht Präsentiertes). Aber auch in Sprachen ohne Artikel vermögen die Sprecher/Hörer natürlich zu unterscheiden, was bereits eingeführt ist und was nicht, weshalb hier die Frage der Determiniertheit nicht minder fundamental ist.

Wird aber nun etwas Neues, noch nicht Genanntes, also Indeterminiertes in den Text eingeführt, so ist damit zugleich eine Art »Existenzaussage « verbunden: Der Satz »Da kommt ein Auto « impliziert zugleich $*$ «Es existiert ein Auto (und kommt da gefahren) «. In den Sätzen, in denen ein Umstandssatz auf ein indeterminiertes Bezugswort folgt, werden diese Umstandssätze prädikativisch auf das implizite * «existieren « bezogen sein: »Ein Mann, der an mit vorbeigeht « < (Es existiert) Ein Mann an mir vorbeigehend/indem er an mir vorbeigeht «. In dieser Art werden die ägyptischen Beispiele zu erklären sein, z.B.

Ebers 51,19-20: »Wenn du siehst einen Mann [ es existiert ein Mann] Schwellungen an seinem Nacken (habend)/(mit) Schwellungen an seinem Nacken «;

Ebers 91,3: »Ein anderes /Heilmittel) für ein Ohr [ es existiert ein $\mathrm{Ohr}]$, indem es fauliges Wasser absondert «.

Ebenso mit Pseudopartizip:

Ebers 49,1-2: » [Es existiert] Ein altes Buch in Öl gekocht «. Urk IV, 663,12: »[Es existiert] Ein Wagen mit Gold beschlagen«.

Die Konstruktion aa) läßt sich also durchaus mit a) und b) vergleichen; es ist wieder dieselbe prädikativische Gebrauchsweise des Pseudopartizips.

Das Pseudopartizip (bzw. generell ein Umstandssatz) vertritt einen Relativsatz allerdings nicht nur nach indeterminiertem Bezugswort, sondern auch noch in zwei anderen Fällen:

— Nach Eigennamen: ${ }^{36}$ Diesen Gebrauch vermag ich nicht zu erklären.

- Wird einem Substantiv eine zusätzliche Qualifikation mit der Bedeutung "ganz« u.ä. beigefügt, verwendet man bemerkenswerterweise im Ägyptischen offenbar grundsätzlich keine attributiven, sondern

36 S. J.P. Allen, The Inflection of the Verb in the Pyramid Texts, Bibliotheca Aegyptia 2, Malibu 1984, $\S 587 \mathrm{~B}$ und allgemein GEG $\S 198,1$ und EAG $\S 1049 ; 1051-52$. 
adverbiale Konstruktionen (s. schon GEG § 317). Von daher erklären sich Ausdrücke wie psdt jeb.tj, ptj tm.tjj (EAG § 595), psdt dmd.tj, jrt tm.tj (GEG § 317). Vgl. auch Sethe, Verbum, II, § 1004 mit zahlreichen Belegen von tm, »vollständig sein « sowie Allen, Inflection, § 586B. Entsprechende Verben, von denen in diesen Fällen ein Pseudopartizip anstelle des zu erwartenden attributiven Partizips gebildet wird, sind $\mathrm{j}^{\mathrm{c}} \mathrm{b}$ (WB I, $40 \mathrm{I}$ ), twt (WB V, $259 \mathrm{~B}$ ), tm (WB V, 304 B), dmd (WB V, 458, D I). Hierher gehören natürlich auch die Adverbialphrasen $r$ 3w.f, $r$ dr.f, mj qd.f, mj qj.f u.ä., die in der Bedeutung »ganz « anstelle eines attributiven Ausdrucks gebraucht werden. Es wäre sicher lohnenswert, diese Erscheinung einmal in größerem Zusammenhang zu untersuchen.

6. Der selbständige Gebrauch des Pseudopartizips nach Fall d) ist von Vergote (p. 349ff.), einem Vorschlag von M.T. Allen folgend, bestritten worden. ${ }^{37}$ Das ist zweifellos nicht aufrechtzuerhalten: Der selbständige Gebrauch der 1. Person dürfte inzwischen unstrittig sein. ${ }^{38}$ In diesem Fall ist das Pseudopartizip natürlich prädikativ, nicht prädikativisch.

7. Eine sehr häufige Funktion des Pseudopartizips ist die als Prädikat im Satzbaumuster des Adverbialsatzes, auf das Subjekt folgend, also das, was Gardiner »pseudoverbal construction « nennt. In den meisten Fällen wird ein solcher Satztyp von jw (GEG § 323), einer Partikel (mk, jst etc., § 324), wnn ( $\$ 326$ ), dem Relativpronomen ( $\$ 328$ ) oder ' $h^{c} . n$ (§ 482) eingeleitet, aber auch Sätze ohne derartige (oder vergleichbare) Einleitungen sind gut bezeugt (s. GEG $\S 322$, Allen, Inflection, § 569 $\mathrm{A} / \mathrm{C}$ und die zahlreichen Belege bei Eigennamen, vgl. LGE $\left.\S 656^{39}\right)$. Wie alle Sätze mit adverbialem Prädikat können auch die mit Pseudopartizip als Prädikat optativisch gebraucht werden, s. z.B. Allen, op. cit., $\S 569$ C, ebenso natürlich als Umstandssatz. Wie oben (§ 4) ausgeführt,

\footnotetext{
37 Zugleich auch einige Fälle des Gebrauchs nach c): Vergote erkennt den »narrativen « Gebrauch des Pseudopartizips nur nach Hilfsverben (wie 'h ${ }^{\circ}$ ) und Partikeln an.

${ }^{38}$ Man vergleiche die Diskussion bei DoRET, Narrative Verbal System, p. 57ff. Ich möchte aber betonen, daß ich in Fällen wie Urk IV, 10,5-6: tnj.kwj ph.n.j j3wj; Hatnub, 14,6: hntj.kwj ph.n.j 3bw, hdj.kwj ph.n.j Mhjt; Mo'alla, IV, 14-5: hnt(w) ph.n.f W3w3t, hd(w) ph.n.f T3-wr die Pseudopartizipien mit Vergote (und Lefebvre) für klare Beispiele von prädikativischem, also untergeordnetem Gebrauch ansehe.

39 Die Erklärung bei Vergote, op. cit., p. 351 zu diesen Namen ist natürlich unhaltbar.
} 
handelt es sich bei Vergotes ${ }^{40}$ Beispiel 28 (= Lebensmüder 86-8: mk b ${ }^{e}$ r rn.j m- ${ }^{-} . \mathrm{k} r$ stj $3 s w \mathrm{~m}$ hrw šmw pt t3.tj) um einen Umstandssatz, nicht um ein Prädikativum in »absoluter « Konstruktion.

Wenn — wie unten gezeigt werden soll — das ägyptische Pseudopartizip historisch und funktional (synchron) nie etwas anderes gewesen ist als ein (konjugiertes) Partizip, stellt sich bei diesem Satztyp folgende Frage:

Wie ist es zu der Kommutabilität des Pseudopartizips mit Präpositionalphrasen (v.a. hr + Infinitiv) gekommen und wieso steht es, wenn es wie hier prädikativ gebraucht wird, nicht vor dem Subjekt, mit anderen Worten, warum verhält sich das Pseudopartizip hier wie ein Adverb?

Die Frage läßt sich nur beantworten, wenn man davon ausgeht, daß die Funktion als Prädikativum, nicht als adverbiales Prädikat, die primäre ist. Etwas ähnliches nimmt übrigens Polotsky für den Satz mit adverbialem Prädikat generell an: »Wahrscheinlich war auch beim Stativ und bei hr plus Infinitiv, als Gliedern der Kategorie der Umstandsformen, die »nähere Bestimmung « das Primäre, und der "Adverbialsatz « wird aus der »adverbialen Satzbestimmung « hervorgegangen sein, so daß, wie C. Brockelmann es präcis ausdrückt, 'das Prädikativ ... den Rang eines Prädikats (gewonnen hat)'. «"1 Eine Möglichkeit wäre also, daß das Prädikativum, das ja (auch) adverbiale Bedeutung hat, zum Prädikat »angehoben « worden wäre. Mir scheint aber ein anderer Weg wahrscheinlicher:

Das ägyptische Pseudopartizip unterscheidet sich morphologisch bekanntlich v.a. darin vom akkadischen Stativ (der der hamitosemitischen Grundform noch sehr nahe stehen muß, s.u.), daß es in allen Formen eine zusätzliche Endung .j aufweist (s. EAG § 576). Diese zusätzliche Endung konnte bis in jüngste Zeit nicht befriedigend erklärt werden. ${ }^{42}$ In GM 117/118, 1990, 181ff. hat F. Kammerzell nun einen

40 Op. cit., p. 347.

${ }^{41}$ Grundlagen des koptischen Satzbaus, II, p. 223.

$42 \mathrm{Vgl}$. etwa LGE $\S 333$, wo eine reichlich abstruse Idee von M. Cohen zitiert wird. Nicht viel besser sind der Vorschlag Diakonoffs (Semito-Hamitic Languages, Moskau 1965, p. 88-9; im Anschluß an Klingenheben), der in .j die »verbal copula jw « sehen möchte und die Ausführungen von KEEs (Ägyptische Schrift und Sprache, HdO 1.1, Leiden 1959, p. 69: »pseudosyllabische Schreibweise «; ähnlich THACKER, The Relationship of the Semitic and Egyptian Verbal Systems, Oxford 1954, p. 120) Daß das .j angehängt wurde, um die Silbe zu schließen und den Abfall der Auslautvokale zu verhindern (so SCHENKEL, Einführung in die altägyptische Sprachwissenschaft, Darmstadt 1990, p. 106), kann ich mir auch nicht vorstellen. Das ist schließlich nirgends sonst im Ägyptischen der Fall. 
ansprechenden Vorschlag gemacht. ${ }^{43}$ Den Schreibungen nach zu urteilen sieht es so aus, daß in den ältesten Texten das Pseudopartizip in der Funktion d) nie eine Endung .j hat, in den anderen Funktionen aber eine haben kann. ${ }^{44}$ Kammerzell möchte nun in diesem .j die auch sonst gut belegbare ägyptische Adverbialendung .j sehen (die schon im Altägyptischen unter unklaren Bedingungen zu .w werden kann), ${ }^{45}$ und das ist ein sehr einleuchtender Gedanke. ${ }^{46} \mathrm{Da} 3$ diese Endung in Texten aus späterer Zeit auch in der 1. Person in der (damals schon sehr reduzierten) Funktion d) auftaucht, ist sicher ganz sekundär und kein ernsthafter Einwand. Folgende Entwicklung scheint möglich (und würde die oben aufgeworfene Frage nach dem Grund für die Verwendung des Pseudopartizips als Adverb beantworten):

1. Die Pseudopartizipien werden von einem bestimmten Zeitpunkt an in ihrer typisch »adverbialen « Funktion als Prädikativum auch äußerlich durch die Adverbialendung .j als Adverbien gekennzeichnet; aus einem Gebrauch »wie ein Adverb« wird ein Gebrauch »als Adverb «. ${ }^{47}$

2. Danach war es für sie — wie für jedes andere Adverb — natürlich problemlos möglich, als Prädikat (Funktion c) in des Satzbaumuster des Adverbialsatzes einzutreten. ${ }^{48}$

43 Seinen Ausgangspunkt, die angeblich so regelmäßige und kaum jemals »defektive « ägyptische Orthographie, beurteile ich allerdings etwas anders. Zu irgendeiner »Ehrfurcht vor der (ägyptischen) Schrift « ist m.E. nicht der geringste Anlaß.

44 Und nach meiner Auffassung ist dann - gegen Kammerzell — in diesen Fällen generell die Endung anzusetzen. Zu den Schreibungen des Pseudopartizips in den Pyramidentexten vgl. jetzt auch meinen Aufsatz in BSEG 15, 1991, 43-56.

45 S. EAG $§ 750-1$; GEG $\S 205$.

46 Erste Ansätze zu einer Erklärung im Sinne von Kammerzell übrigens schon bei ERMAN, Ägyptische Grammatik ${ }^{4}$, § 325 und 331a.

47 Das wäre ein hübsches Beispiel für das Prinzip »form follows function«, auf das Kammerzell in seinem zitierten Aufsatz solchen Wert legt.

Die formale Kennzeichnung der Pseudopartizipien in adverbialem Gebrauch als Adverbien hängt vielleicht auch damit zusammen, daß ein Homonymenkonflikt vermieden werden sollte: Wenn man die Pseudopartizipien nach dem Vorbild des akkadischen Stativs ansetzt, ergäbe sich für die 1. und 2. Person z.B. *sadmáku, sadmáta, sadmáti > *sadmák, sadmắt, sadmắt > *sad̄mắk, sadmắt, sad̄mắt, und điese letzten Formen wären identisch mit der ș̄m.f-Form der 2. Sg. m./fem. oder, besser gesagt, mit einer sd̄m.fForm (die in verschiedenen Funktionen belegt ist, vgl. J. Osing, Der spätägyptische Papyrus $B M$ 10808, $\ddot{A} A 33$, Wiesbaden 1976, p. 33-6; 167-70 [247], wenn auch natürlich nicht sicher ist, wie alt diese vokalisch rekonstruierbaren Formen tatsächlich sind, s. Osing, op. cit., p. 169-70). Nach Antritt eines .j ändert sich natürlich die gesamte Wortstruktur: Die dann entstehenden Formen sind im Ägyptischen unverwechselbar.

48 Auf die besondere Fähigkeit passiver Partizipien, neue Konjugationsschemata zu bilden, die auch hier wieder sichtbar wird, werde ich unten noch zurüickkommen. 
3. In der Funktion d) wird aber natürlich kein .j angehängt, da es sich hier ja nicht um ein adverbial gebrauchtes Pseudopartizip handelt. ${ }^{49}$

Nur in dieser Reihenfolge ergibt sich eine logische Entwicklung. Wenn Polotsky einmal sagt »It was presumably through its contrasting association with this particular prepositional phrase [hr + Inf.] that the Pseudo-Participle acquired its surprising property of patterning with adverbial expressions generally $\ll^{50}$, werden m.E. die Dinge auf den Kopf gestellt.

8. Überblickt man Ableitung, Bedeutung und Funktion des ägyptischen Pseudopartizips, so spricht eigentlich alles dafür, daß es sich einfach um ein (konjugiertes) passives Partizip handelt, in bestimmten Fällen genauer gesagt ein adverbiales konjugiertes passives Partizip. Das läßt sich an folgenden Punkten ablesen:

8.1. Die Bedeutung des Pseudopartizips ist passiv bei transitiven Verben (zu den Aktiv-Fällen s.u.), resultativ bei intransitiven und deskriptiv bei nichtfientischen (Eigenschafts-) Verben. Das ist genau die Bedeutung, die man bei einem »Zustandspartizip" (bzw. Partizip passiv) erwarten sollte. Dasselbe gilt übrigens für das akkadische »Verbaladjektiv «. ${ }^{51}$

\section{Exkurs zur Terminologie:}

Wenn ein Verbaladjektiv, das bei transitiven Verben passive Bedeutung hat, auch von intransitiven und nichtfientischen Verben gebildet werden kann, ist die Bezeichnung "passives Partizip« sicher nicht ganz glücklich. Die Assyriologen sprechen deshalb einfach von »Verbaladjektiv « (obwohl das nicht besser ist, da auch pāris(u) ein deverbales Adjektiv ist!), A. Gai hat »non-active participle « vorgeschlagen (ZDMG 136, 1986, 14 [4]), O.E. Ravn (ArOr 17,2, 1949, 303)»Participium II « (für das akkadische parsu). In Wirklichkeit wird es sich einfach um ein altes Partizip Perfekt handeln (oder, um das Reizwort »Perfekt « zu vermeiden, ein Vergangenheitpartizip im Gegensatz zum

${ }^{49}$ Zum (angeblich?) selbständigen Gebrauch der 2. und 3. Person s.u., Anm. 76. Beim sogenannten »optativischen « Pseudopartizip handelt es sich natürlich nicht um eine wirklich selbständige (nichtadverbiale) Funktion.

50 Collected Papers, p. 328.

51 S. Huehnergard, JNES 46, 1987, 225. 
präsentischen pāris): Ist eine Handlung, ein Vorgang, ein Zustand vollendet, resultiert er in einer Eigenschaft (Eigenschaftsverben: deskriptiv), einem Zustand (intransitive Verben: resultativ) und das Objekt ist von der Handlung getroffen (transitive Verben: passiv). Es ist ja kein Zufall, daß viele Sprachen zwei Partizipien mit einer Kombination präsentisch/aktiv/Vorgang vs. perfektisch/passiv/Zustand haben.

Allerdings gibt es natürlich Unterschiede: Z.B. gebraucht das Ägyptische das Pseudopartizip dort, wo man im Deutschen oder Lateinischen ein Partizip Präsens/aktiv hätte: »Ich traf ihn schlafend an « wäre ägyptisch etwa *gmj.n.j sw qd(w). Auch das Akkadische gebraucht sein passives Partizip (Verbaladjektiv) öfter dort, wo man in europäischen Sprachen eher ein aktives/präsentisches Partizip erwarten würde. Bei einem präsentisch/gleichzeitigen Zustand ist im Deutschen oder Lateinischen (etc.) offenbar die temporale Seite wichtiger, im Ägyptischen (oder Akkadischen) die »zuständliche «.

Die verschiedenen Bedeutungsmöglichkeiten des Pseudopartizips, ebenso seine Affinität zur Vergangenheit, erklären sich also mühelos, nimmt man an, daß es sich um ein altes Partizip Perfekt (passiv) handelt, während dies alles bei einer finiten Verbalform ziemlich merkwürdig wäre.

Es ist wirklich ein Unding, daß unter »perfektischem Partizip « in der Ägyptologie das präsentische Vorgangspartizip sādim geführt wird! Polotsky hat die verschlungenen Pfade in der Verwendung der Bezeichnungen perfektisch/perfektiv und imperfektisch/imperfektiv nachgezeichnet. ${ }^{52} \mathrm{Da} 3$ diese Bezeichnungen, auf die Partizipien angewendet, »harmlos « seien und »bisher keinen Schaden angerichtet « hätten (p. 769), würde ich nicht unterschreiben: Es hat dazu geführt, daß in den heutigen Handbüchern die Lage so dargestellt wird, als ob das mrr-Partizip präsentisch und das nichtgeminierende Partizip perfektisch sei! Es ist natürlich nicht auszuschließen, daß das mrr-Partizip, das ja an sich eine andere Aktionsart bezeichnet,,$^{53}$ das alte Vorgangspartizip sādim in einigen Verwendungsweisen als »normales « Partizip Präsens verdrängt hat (z.B. beim »participial statement «). Eine neue unvoreingenommene Untersuchung der Bedeutungssphären der Partizipien würde sich aber

52 Studies in Egyptology (Fs Lichtheim), Jerusalem 1990, II, 768-72.

53 S. zuletzt Allen, Inflection, p. $421 \mathrm{ff}$., der dort eine wichtige Entdeckung von Schenkel bestätigt und ausgebaut hat. 
empfehlen. Vorläufig habe ich den Verdacht, daß man den »perfektischen « Partizipien (sādim) gewisse Eigenschaften nur deshalb unterstellt, weil sie eben den Namen »perfektisch « tragen.

8.2. Bestimmte Suppletivverhältnisse des Pseudopartizips mit Partizipien deuten gleichfalls darauf hin, daß auch das Pseudopartizip eine Art Partizip ist.

- Es ist festgestellt worden, daß im AR das selbständige Pseudopartizip der 1. Person (in aktivischer Bedeutung; Funktion d) mit einer meist als »perfektisches sdm.f « bezeichneten Form eine Art Paradigma bildet, aber nur, wenn diese Form ein substantivisches (kein pronominales) Subjekt hat. ${ }^{54}$ Es ist wohl kein Zufall, daß hier die 1. Person und die 3. Person mit substantivischer Besetzung als Subjektsbesetzung eine Verbindung eingehen bzw. von einem vollständigeren Paradigma übriggeblieben sind: Nur in diesen Fällen ist es auch ohne Kontext klar, auf wen das Subjekt verweist. ${ }^{55}$ Satzinger hat nun (fragend) vorgeschlagen, ob es sich hier vielleicht gar nicht um ein (verbales) sdm.f handelt, sondern einfach um das prädikativ gebrauchte Partizip. ${ }^{56}$ Ich halte diese Idee für sehr überzeugend; zu Folgerungen daraus, s.u., § 10.

— In PT 495b (W) hat die ältere Version ein Pseudopartizip 1. Sg.: jrh.k, die neuere, in die 3. Person umgesetzte aber ein prädikatives Partizip: jrh sw. Im Licht dieser Stelle ist wohl auch § 1434a zu verstehen, wo dem Pseudopartizip jrh.k (rn.k) in P (Z.646 + 715) ein jrh NN (rn.k) in M entspricht: Auch bei $M$ dürfte es sich um ein prädikatives Partizip, nicht um ein sdm.f handeln.

- Der Adjektivsatz ist bekanntlich auf die 2. und 3. Person beschränkt, vgl. GEG § 137. Die entsprechende Suppletivform für die 1. Person ist aber kaum der Nominalsatz mit jnk ${ }^{57}$ sondern das Pseudopartizip,

54 S. Osing, OrNS 46, 1977, 166ff.; DORET, The Narrative Verbal System of Old and Middle Egyptian, Genf 1986,, p. 21ff.; 57ff. Dieses Paradigma ist als solches von Osing entdeckt worden; er möchte allerdings auch Pseudopartizipien in der 3. Person zulassen. S. dagegen Doret, op. cit., p. 61; 63; 65, der die entsprechenden Belege als untergeordnet interpretiert. Zu den Unterschieden bei Osing und Doret vgl. die Diskussion bei Schenkel, AfO 35, 1988, 237ff.

55 Und insofern hätten Osing und Doret beide recht: Sowohl der Unterschied 1. und 3. Person als auch der Unterschied substantivisches/pronominales Subjekt sind relevant.

56 WZKM 79, 1989, 212.

57 So z.B. Schenkel, AfO 35, 1988, 238. 
s. GEG § 312, Punkt 4: Sowohl ein Adjektivsatz (nfr sw/Subst.) als auch das prädikative Pseudopartizip eines Adjektivverbs haben beide die gleiche Bedeutung, nämlich das einfache »Zusprechen einer Eigenschaft «. Demgegenüber hat ein Nominalsatz des Typs jnk nfr eher die Bedeutung »Gleichsetzung mit einem Typus «.

- In der Funktion a) und c) kann das Pseudopartizip 3. fem. durch ein prädikativ gebrauchtes Adjektiv/Partizip + folgendes Subjektspronomen s(j) ersetzt werden, s. GEG § 374 und Grapow, ZÄS 71, 1935, $52 \mathrm{ff}$. Man hat den Eindruck, als ob die alte, ägyptisch an sich gar nicht mehr durchschaubare Bildung prädikatives Partizip + Subjektspronomen hier erneuert worden sei. ${ }^{58}$

- Wenn Edel ${ }^{59}$, wie anzunehmen ist, recht darin hat, daß die altererbte Endung des Pseudopartizips in der 1. Plural nur einmal (als nw) belegt ist, die übliche Endung (geschrieben wn oder wjn) aber tatsächlich die »Admirativpartikel « wj + enklitisches n »wir « repräsentiert, hätte man in der Tat eine Formation prädikatives Partizip + wj + Subjektspronomen im aktuellen Paradigma des Pseudopartizips.

- Lefebvre ${ }^{60}$ hat auf einige Fälle hingewiesen, in denen ein passives Partizip an einer Stelle steht, wo man eigentlich ein Pseudopartizip erwarten sollte.

8.3. Der Gebrauch einer Form als Prädikativum ist zwar in keiner Weise auf Partizipien beschränkt, aber doch gerade bei ihnen besonders häufig. Man vergleiche nur die Verhältnisse im Griechischen und Lateinischen.

8.4. Die Bildung neuer Konjugationsmuster, wie es durch den Eintritt des Pseudopartizips in die »pseudoverbale Konstruktion « > Präsens I geschieht, ist ganz besonders typisch für passive Partizipien. Neben der Entwicklung vom »Stativ « (= Nominalsatz mit Partizip) zum »Perfekt « im Semitischen (s.u.) vergleiche man die Geschichte der romanischen und germanischen Sprachen mit einer Fülle von Beispielen!

8 Verrückterweise ausgerechnet in der 3. Person, wo ja die ursprünglich Formation gar kein eigenes Subjektspronomen hat!

59 EAG § 574aa; ebenso Allen, Inflection, § 564D.

${ }^{60}$ LGE $\$ 357$. 
9. Die Herkunft des ägyptischen Pseudopartizips, seine historische Identität mit der Form, die im Akkadischen als »Stativ « erscheint, beweist, daß das Pseudopartizip von einem passiven Partizip »abstammt «. Diese Verwandtschaft ist so augenfällig, daß sie kaum eines Beweises bedarf. Es ist zweifellos so, daß der akkadische Stativ der gemeinsamen Vorform am nächsten steht, ${ }^{61}$ da die Bildung dieser Form allein hier noch transparent ist. ${ }^{62}$ Die innersemitische Entwicklung zum »Perfekt « braucht hier nicht weiter zu interessieren, da sie für den Vergleich mit dem Ägyptischen belanglos ist. ${ }^{63}$

Der akkadische Stativ war bisher meist als »konjugiertes Nomen « eingestuft worden ${ }^{64}$ dessen Basis — abgesehen von der innerakkadischen Sonderentwicklung, die auch die »Konjugation « von Substantiven ermöglicht (s.u.) — von »Verbaladjektiven « gebildet wird: »Beim Verbum ist der St[ativ] das konjugierte Verbaladj[ektiv] ... wie dieses ein deklinierter St[ativ] ist; beide Kategorien sind also identisch. ${ }^{65}$ Das »Verbaladjektiv « ist aber nichts anderes als das passive Partizip des Akkadischen. ${ }^{66}$

Die Formation des Satztyps, der in der Assyriologie als »Stativ« bezeichnet wird, ist zum ersten Mal überzeugend von G. Buccellati dargestellt worden ${ }^{67}$ und in verbesserter Form dann v.a. in zwei brillanten Beiträgen von J. Huehnergard. ${ }^{68}$ Einige in unserem Zusammenhang interessierende Ergebnisse:

${ }^{61} \mathrm{Zu}$ dem historischen Abkömmling in den Berbersprachen s. Rössler, ZDMG 100, $1950,481 \mathrm{ff}$.

62 Vgl. auch Polotsky, Collected Papers, p. 327.

63 Aber auch im westsemitischen Perfekt sind Reste der nominalen Entstehung noch nachweisbar, s. PRIEBATSCH, "Der Weg des semitischen Perfekts", Ugarit-Forschungen $10,1978,337-47$.

64 Von SODEn, Grundriß der akkadischen Grammatik, § 77a.

65 Op. cit., § 77,d (Hervorhebung im Original).

Wenn F.R. KRaus, Nominalsätze in altbabylonischen Briefen und der Stativ, Amsterdam 1984, p. 20, sich fragt »Was bedeutet der erste Bestandteil des Stativs und warum ist er in allen Formen unveränderlich der gleiche? « und »In welcher Beziehung steht der zweite Bestandteil des Stativs zum ersten? «, so heißt das doch, den Wald vor lauter Bäumen nicht zu sehen. Huehnergards unten herangezogene Aufsätze sind nicht zuletzt als Reaktion auf Kraus' merkwürdige Ansicht entstanden.

${ }^{66}$ S. Goetze, JAOS 62, 1942, 5b und v.a. A. GAI, "The Non-Active Participles in the Ancient Semitic Languages «, ZDMG 136, 1986, 8-14.

67 "An Interpretation of the Akkadian Stative as a Nominal Sentence«, JNES 27, 1968, 1-12.

68 „On Verbless Clauses in Akkadian «, Zeitschrift für Assyriologie 76, 1986, 218-49; "' 'Stative', Predicative Form, Pseudo-Verb «, JNES 46, 1987, 215-32.

Man vergleiche auch KIENAST, "Der sogenannte 'Stativ' des Akkadischen" in: ZDMG, Suppl. IV, 1980, 84-6 mit Parallelen aus jüngeren semitischen Sprachen. 
— Der »Stativ « ist kein verbales Konjugationsmuster, sondern einfach ein Nominalsatz, bestehend aus einem Nomen (ursprünglich immer ein $\gg$ Verbaladjektiv«) im endungslosen status praedicativus mit einem enklitisch folgenden Subjektspronomen. Das gilt wohlgemerkt nicht (nur) für die Genese dieser Form im Hamitosemitischen, sondern synchron für das Akkadische! Huehnergard ${ }^{69}$ möchte denn auch ganz konsequent und einleuchtend den »Stativ « völlig aus der Verbalmorphologie einer deskriptiven Grammatik des Akkadischen verbannt wissen: Es reiche aus, den prädikativen Status des Nomens in der Nominalmorphologie aufzuführen und die enklitischen Allomorphe des Subjektspronomens bei der Behandlung der Pronomina; dazu in der Syntax die Konstruktion Nomen im status praedicativus + enklitisches Pronomen als einen Fall unter den Nominalsätzen (mit den entsprechenden Konstruktionsregeln).

— Die Bedeutung des »Stativs « (passiv bei transitiven Verben, resultativ bei intransitiven, »deskriptiv « bei Zustandsverben) beruht allein auf der Bedeutung des Verbaladjektivs (Partizip passiv), nicht auf der einer Konstruktion oder Form »Stativ «!

- Der Einschluß von Substantiven in diese Konstruktion (z.B. šarrāku) ist eine innerakkadische Innovation. Das zeigt die Tatsache, daß es dies nur im Akkadischen gibt, ${ }^{70}$ ebenso wie die Datierung der Belege. ${ }^{71}$

- Stative von transitiven Verben, die aktive Bedeutung haben, ${ }^{72}$ was v.a. bei einer kleinen Gruppe von Verben (șabātu, leqû, mahăru und našû) häufig ist, sind durch analogische Ausdehnung entstanden ${ }^{73}$ und kein Nominalsatz (bzw. Adjektivsatz), da eine nominale Basis entsprechender Bedeutung fehlt. Huehnergard spricht deshalb von einem »Pseudoverb «. Es handelt sich auch hier zweifellos um eine innerakkadische Entwicklung, die für komparatistische Zwecke zu vernachlässigen ist.

Die Ausführungen von Buccellati und Huehnergard sind in mehrfacher Hinsicht für das Ägyptische (und generell für das Hamitosemitische) von Bedeutung:

69 JNES 46, 230-1.

70 S. ZA 76, 239, n. 77-78.

71 S. Kraus, op. cit., p. 14ff.; JNES 46, 223, n. 23.

72 Also der »permansive « bei Rowton, JNES 21, 1962, 233-303.

73 Von Huehnergard in Anlehnung an T.O. Lambdin sehr überzeugend erklärt, s. JNES 46, p. 228-9. 
— Die Interpretation des »Stativs « als prädikatives Zustandspartizip + Subjekt muß natürlich auch für das Hamitosemitische zutreffen (oder, vorsichtiger ausgedrückt, für alle hamitosemitischen Sprachen, die Abkömmlinge dieser Formation haben), eben weil seine Bildung im Akkadischen noch vollkommen durchschaubar ist und es sogar möglich ist, die Erscheinung nur mithilfe der allgemeinen akkadischen Morphologie und Syntax, ohne Ansatz einer eigenständigen Kategorie »Stativ «, gerecht zu werden. Wenn eine Form wie der "Stativ «, bestehend aus einem Verbalnomen als Kern und pronominalen Affixen, in mehreren verwandten Sprachen belegt ist, und in der weitaus ältesten davon diese Formation durchschaubar ist - ihre Bestandteile gehören zum normalen morphologischen Inventar und die Syntax ihrer Komposition entspricht der der übrigen Sprache dann gibt es m.E. nur eine naheliegende Folgerung: Dieser in der alten Sprache belegte Zustand ist der Urzustand. ${ }^{74}$

- Daraus folgt, daß die Formation, die im Ägyptischen das »Pseudopartizip« ergeben hat, im Hamitosemitischen noch gar nicht zum Verbalsystem gehörte. Deshalb ist es nicht weiter verwunderlich, wenn die semitische »Suffixkonjugation « im Ägyptischen vorhanden ist, die »Präfixkonjugation « hingegen nicht. Das Ägyptische wird eben nach seiner Ausgliederung sein Verbalsystem innoviert haben, während das Syntagma, das dann zum Pseudopartizip (bzw. semitisch zur »Suffixkonjugation «) wurde, noch ein Nominalsatz (Adjektivsatz) war.

- Die aktivische Bedeutung transitiver Verbstämme im Stativ ist (ebenso wie die Ausdehnung der Formation auf alle Nomina) eine innerakkadische Entwicklung, also nicht in der Grundsprache vorhanden..$^{75}$

- Das heißt aber, daß der aktivische Gebrauch des Pseudopartizips im Ägyptischen ebenfalls eine innerägyptische Entwicklung sein muß

74 O. Rössler hat übrigens die hamitosemitische »Suffixkonjugation « für die Ursprache als »konjugiertes Nomen oder Partizipium « angesetzt, s. ZDMG 100, 1950, 466. Wenn, wie anzunehmen, das Akkadische in diesem Punkt weitestgehend den ursprünglichen Zustand bewahrt hat, wäre der von Rössler vermutete Stand erst im Altägyptischen erreicht.

75 Die anderslautende These von H.P. Müller, SÄK 11, 1984, 365ff. war ohnehin wenig überzeugend und dürfte durch die Ausführungen von Huehnergard und Kraus (s.o.) vollends unhaltbar geworden sein. 
(und überhaupt nichts mit »Perfekt« zu tun hat!). Ohnehin sind ja die akkadischen und ägyptischen Aktiv-Formen des Stativs/Pseudopartizips in jeder Hinsicht unvergleichbar.

10. Im Ägyptischen ist die Bildung des Pseudopartizips nun aber nicht mehr transparent und schon von daher ist es klar, daß sie sich in besonderer Weise weiterentwickeln konnte. Die teilweise Spezialisierung auf die Funktion »Prädikativum «, der Antritt der Adverbialendung . $\mathrm{j}$ in dieser adverbialen Funktion und der dadurch mögliche Eintritt in das Schema des Adverbialsatzes sind oben dargelegt worden.

Wie verhält es sich nun mit dem ursprünglichen, im Akkadischen (und ebenso wohl auch in der Grundsprache) absolut dominierenden Gebrauch als Satz (d.h. in der Funktion d)? Wie oben erwähnt, ist er im Ägyptischen auf die 1. Person Sg. beschränkt, ${ }^{76}$ wo er in alten Texten mit dem sogenannten "perfektischen « sdm.f (nur in der 3. Person mit nominalem Subjekt) eine Art Paradigma bildet. Nach Satzingers ansprechender Vermutung (s.o.) dürfte dieses angebliche sdm.f nichts anderes sein als ein prädikatives Partizip. Es ließe sich folgende Entwicklung denken:

In einem (nicht mehr in den Texten greifbaren) sehr frühen Zustand des Altägyptischen wird das Pseudopartizip in seiner alten prädikativen Funktion (wie im Akkadischen) mit nominalem und pronominalem Subjekt als mehr oder weniger vollständiges Paradigma vorhanden gewesen sein, d.h. als prädikatives Zustandspartizip + nominales Subjekt und prädikatives Zustandspartizip + affigiertes Subjektspronomen.

Als dann zu einem unbekannten Zeitpunkt die spezifisch ägyptischen Silbenstrukturgesetze in Kraft traten (in offener Tonsilbe ausschließlich Langvokal, in geschlossener Tonsilbe ausschließlich Kurzvokal), war eine Uminterpretation der Form der 3. Person unvermeidlich: Ein sắdim ist nach den genannten Silbenstrukturgesetzen nicht möglich, daraus mußte sắdim werden. Ein sấdim aber ist (auch im Ägyptischen) die normale Form des aktiven Partizips. Das heißt aber, ein Satz mit prädikativem sấdim wäre notwendig als aktiv verstanden worden. Von

76 Nach Allen, Inflection, $\S 311$ kommt dieser Gebrauch in den PT bei intransitiven Verben auch in der 2. und 3. Person vor. Das ist (zumal für die 2. Person) nicht ausgeschlossen (und im Prinzip spricht auch gar nichts dagegen), die Belege sind aber nicht wirklich zwingend und können auch anders interpretiert werden. Vgl. im einzelnen jetzt BSEG 15, 1991, 55-6. 
der (häufigen) 3. Person wird sich diese aktivische Bedeutung analogisch auf die anderen Personen ausgedehnt haben, und genau das wird der Grund dafür sein, daß das Pseudopartizip (der 1. Person) eben in den Fällen, wo es ein Suppletivverhältnis mit einem sdm der 3. Person bildet, und nur in diesem Fall, aktiv ist. ${ }^{77}$

Als das Paradigma dann nach dem Alten Reich auseinanderfiel, d.h. der Zusammenhang von prädikativem Partizip und Pseudopartizip 1. Person nicht mehr deutlich war, ist auch der aktive Gebrauch der 1. Person ausgestorben (abgesehen von rh, das sich aber in vieler Hinsicht abweichend verhält und in allen Personen des Pseudopartizips aktivische Bedeutung hat). Der unabhängige Gebrauch des Pseudopartizips in der 1. Person bleibt allerdings (im Mittelägyptischen) bis in die Spätzeit erhalten, aber nur - wie in allen anderen Funktionen des Pseudopartizips - in passiver Bedeutung.

Aus einem ursprünglichen Nominalsatz (bzw. Adjektivsatz) mit einem Partizip Perfekt passiv als Prädikat ist also im frühesten Ägyptisch, als die Konstruktion nicht mehr durchschaubar war, ihre

77 Das betrifft allerdings nur die Privat- und Königsinschriften des AR, d.h. das in den Urk I gesammelte Material. In den PT kommen in der Funktion d) (abgesehen von dem Sonderfall rh) nur intransitive Verben vor, s. Allen, Inflection, $\$ 590$.

Es gibt einige Fälle, wo aktiv-transitive Pseudopartizipien in der 2. Person und/oder in adverbialer Funktion vermutet worden sind:

1. Eine beigeschriebene Rede bei von Bissing, Die Mastaba des Gem-ni-kai, Berlin 1905, pl. XXIII ( = ERMAN, Reden, Rufe und Lieder, APAW 1918, Berlin 1919, p. 50): jdd tw jn jw.k wn.tj mdw pw nfr n š wird in EAG $\S 590$ B übersetzt: »Rede doch! Gehst du (etwa) an diesem schönen 'Stock des Sees' vorüber? « Eine Übersetzung ohne Objekt ist aber sicher vorzuziehen: »Sprich doch! Gehst du (etwa) vorüber? Dies ist ein schöner 'Stock des Sees'! « Vgl. auch Silverman, Interrogative Constructions with jn and jn-jw in Old and Middle Egyptian, Bibliotheca Aegyptia 1, Malibu 1980, p. 35, n.199.

2. Die Belege von jdd.t aus den PT, die Edel (op. cit., § 594) als optativische aktive Pseudopartizipien deutet, sind mit Allen, Inflection, $\S 550$ sicher als sdm.tj-Passive zu verstehen.

3. Edels Belege (loc. cit.) für wnh.t(j) dt.k »bekleide dich mit deinem Leib (PT 1300c; 2119; 224d) werden von Kammerzell, GM 117/118, 1990, 188, n. 15 ebenfalls als Fälle von sdm.tj-Passiv aufgefaßt. Möglich wäre auch, dt.k als Apposition zu verstehen: »sei bekleidet, (nämlich) dein Leib «.

4. Als einziger problematischer Beleg bleibt PT $2110 \mathrm{~d}$ : (b3.k $\mathrm{m}$ hrw sd3.k m grh ntr is nb št) wd.tj mdw ntrw shm js hnt [ $\left.{ }^{\circ} \mathrm{nhw} w\right]$, der allgemein als aktiv-transitiv verstanden wird, s. EAG § 590ß; Allen, Inflection, $\S 592$; Kammerzell, op. cit., p. 188, n. 15; Faulkner, Pyr., p. 299 und 300, n.18. Trotz dieser allgemeinen Übereinstimmung scheint mir eine Auffassung als sdm.tj-Passiv (als nachgestellter Temporalsatz) dem Sinn nach keineswegs unmöglich oder auch nur besonders gezwungen. Nur einen einzigen Beleg für diese Bedeutung zu haben (s. Allen, Inflection, §592) wäre viel unwahrscheinlicher. 
Kompositionsglieder nicht mehr als einzelne zum normalen morphologischen Bestand gehören, ein konjugiertes Partizip geworden, das folgendermaßen gebraucht wird:

- Prädikativ am Satzanfang, auf die 1. und (mit nominalem Subjekt) 3. Person beschränkt und durch lautgesetzliche Änderungen als aktivisch reinterpretiert (Funktion d);

— Als prädikativisches (»adverbiales «) Partizip: In dieser Verwendung wird die Adverbialendung .j (>.w) angehängt und aus einem Gebrauch wie ein Adverb wird ein Gebrauch als Adverb (Funktion a): Hier handelt es sich um die Fälle, wo das Pseudopartizip bisher als »Umstandssatz ohne eigenes Subjekt « betrachtet wurde.

- Als Prädikativum mit vorausgehendem substantivischen Begleiter in einer »absoluten « Partizipialkonstruktion (Funktion b): Dazu gehört die Mehrzahl der Fälle, die bislang als Umstandssätze mit prädikativem Pseudopartizip bestimmt wurden.

- Als adverbiales Prädikat in Haupt- und Nebensatz (Funktion c: keine Änderung gegenüber dem üblichen Verständnis).

Diese Sicht der Dinge wird den Belegen ebensogut gerecht wie die bisherige, hat ihr gegenüber aber noch zusätzliche Vorzüge: Sie vermag die Gebrauchsweisen aa), bb) und d) ebenso zu erklären wie die Entstehung der Funktion des Pseudopartizips als adverbiales Prädikat, und generell lassen sich Genese und Funktion dieser Form in einen sinnvollen historischen Zusammenhang bringen.

11. Auch die weitere Geschichte des Pseudopartizips, seine Entwicklung im Neuägyptischen, Demotischen und Koptischen, zeigt die partizipiale Bedeutung des Pseudopartizips deutlich. Es bleibt seiner Bedeutung nach immer ein Zustandspartizip und wird im Koptischen nur noch prädikativ (Funktion c) gebraucht, während im Neuägyptischen auch Funktion a) noch vorhanden ist.

Im Koptischen kann von einem Verb (grundsätzlich) nur noch eine Qualitativ-Form gebildet werden, die entweder auf die 3. Person maskulin oder feminin zurückgeht. Schon im Neuägyptischen beginnt sich die Entwicklung deutlich abzuzeichnen, indem dort Formen der 3. Person mask. oder fem. für alle Personen eintreten können. ${ }^{78}$ Ein Zusammenfall 
der Personalendungen wäre aber bei einem finiten Verb etwas Unerhörtes! Daß es hier geschieht, erklärt sich eben daraus, daß die Form nie als etwas anderes denn als Partizip empfunden worden ist. ${ }^{79}$

12. Es handelt sich beim ägyptischen Pseudopartizip also um eine deverbale Nominalform, um ein Zustandspartizip, das während eines Abschnitts seiner Geschichte eine einzige Eigenschaft des finiten Verbs, die Flexion nach Personen, angenommen hat. Ein »konjugiertes Partizip « ist auch keineswegs etwas besonders unübliches, sondern auch in anderen Sprachen durchaus belegt. So sind etwa in den finno-ugrischen Sprachen konjugierte Partizipien (und andere Verbalnomina) sehr verbreitet (freundliche Auskunft von Dr. R.-P. Ritter, FU Berlin). Auch z.B. im grönländischen Eskimo gibt es sogenannte transitive bzw. intransitive Partizipien, die nach Numerus und Person flektiert werden. ${ }^{80}$ Dennoch aber ist das Pseudopartizip nach Herkunft, Bedeutung, Funktion und Entwicklung niemals etwas anderes gewesen als ein Zustandspartizip und zweifellos während seiner gesamten langen Geschichte - vom Hamitosemitischen bis zum Koptische - immer als Partizip empfunden worden. ${ }^{81}$

\author{
Ägyptologisches Seminar \\ Freie Universität Berlin \\ Altensteinstr. 33 \\ D-14195 Berlin
}

\footnotetext{
${ }^{79} \mathrm{Da}$ »das koptische Qualitativ wirklich zu einer Art Particip geworden ist «, ist auch Ermans Ansicht (Neuägyptische Grammatik ${ }^{2}, \S 327$ ), und an dieser Aussage ist nur das " geworden « $\mathrm{zu}$ beanstanden.

80 S.H. Walter, Studien zur Nomen-Verb-Distinktion aus typologischer Sicht, München 1981 , p. $42 \mathrm{ff}$.

81 Übrigens hat schon W. Golenischeff das Pseudopartizip als »konjugiertes Partizip«, das es im Alt- und Mittelägyptischen ja ist, betrachtet (leider ohne Begründung), s. Le Conte du naufragé (BdE 2, 1912), p. 30, 41, 43, 45 u.ö.: »participe à flexion(s)«, etwas ausführlicher in seinem Aufsatz "Quelques remarques sur la syntaxe égyptienne" in: Fs Champollion (Paris 1922), p. 685ff. (»participe conjugable«, p. 688). Eine ausführliche Würdigung von Golenischeffs Ansichten und seiner Kontroverse mit den Thesen der »Berliner Schule « gibt Struve in: Fs Golenischeff, p. 31ff.
} 